\title{
Postmortem cerebrospinal fluid analysis in a general intensive care unit
}

\author{
Mansour Hasani,, ${ }^{1}$ Mohammad Ali Sahraian,, ${ }^{2}$ Mahmoud Motamedi, ${ }^{3}$ Kamran Mostehaghan ${ }^{4}$
}

\begin{abstract}
논
Background and Aim: Infection is a common problem in intensive care medicine and many patients are treated aggressively and empirically with broad-spectrum antibiotics. Meningitis may be missed in intensive care unit (ICU) because some of these patients have fever and altered mental status from another illness and may be treated with impression of sepsis. In order to identify undiagnosed meningitis in ICU admitted patients, postmortem CSF analysis was performed immediately after death. Subjects and Methods: During 1 year, 43 patients underwent lumbar puncture after death. Samples were collected and analysis for cells, sugar and protein concentrations were obtained. Demographic characteristics and diagnosis of the patients were recorded and data analysis was performed by SPSS version 11.5. Results: CSF was completely normal in $62.8 \%$ of the patients and it was compatible with meningitis in two of them. Both of them had admitted with impression of sepsis and had another source of infection. Considering a subgroup of the patients who admitted to ICU with impression of sepsis, they were statistically significant. There was no significant difference between surgical and nonsurgical patients. Conclusions: Our findings suggest that meningitis may be missed in ICUs, and more liberal use of CSF analysis should be considered in critically ill patients, especially those who referred with impression of sepsis.
\end{abstract}

Key Words: Cerebrospinal fluid, meningitis, critical care, missed diagnosis

\section{Introduction}

The emergence of fever and mental status changes during the management of a critically ill patient is an extremely common and challenging problem. Many infectious and noninfectious etiologies should be considered in differential diagnosis of such patients. Early diagnosis and proper treatment is mandatory because most of these patients cannot withstand progression of a septic process.

Inadequate antimicrobial treatment of infections among

\section{From:}

${ }^{1}$ Associate Professor of Anesthesiology, ${ }^{2}$ Assistant Professor of Neurology,

${ }^{3}$ Associate Professor of Neurology, ${ }^{4}$ Resident of Anesthesiology, Sina Hospital,

Tehran University of Medical Sciences, Hassan Abad Square, Imam Khomini

Street, Tehran, Iran

Correspondence:

Mohammad Ali Sahraian,

E-mail: msahrai@sina.tums.ac.ir patients requiring intensive care is an independent determinant of hospital mortality. ${ }^{[1]}$ Meningitis is one of the causes of fever and mental status changes in patients admitted in intensive care units (ICUs) and may be easily missed because of another underlying illness. Moreover, in some cases requiring ICU care, lumbar puncture is contraindicated because of increased intracranial pressure, for example, patients with intracerebral hemorrhage or brain tumor. Such cases are usually treated with broad-spectrum empirical antibiotics.

This study was performed to identify undiagnosed meningitis with cerebrospinal fluid (CSF) examination immediately after death.

\section{Materials and Methods}

This prospective study was performed in the Sina Hospital, a major teaching hospital of Tehran University of

Free full text available from www.ijccm.org 
Medical Sciences, from June 2003 to June 2004. During this study all patients who deceased $72 \mathrm{~h}$ after admission in ICU underwent lumbar puncture immediately after death. CSF analysis for total and differential white blood cells, red blood cells, and protein and glucose concentrations were performed. A sample for CSF culture was sent too.

Meningitis was defined as CSF white-blood-cell (WBC) counts of greater than 5 cells $/ \mathrm{ml}$, decreased glucose, and increased protein concentrations. Demographic parameters such as age, sex, reason of ICU admission, length of ICU stay, cause of death, type of antibiotics receiving during admission, physical findings such as fever and neck stiffness, and past medical history were recorded for each patient. An informed consent was obtained from the family before doing lumbar puncture and those who did not have consent from the family were excluded from the study.

Patients with the diagnosis of meningitis and those who deceased in the first 3 days of ICU admission were also excluded. After data gathering, statistical analysis was done by SPSS version 11.5.

\section{Results}

During 1 year, 52 patients expired in our general ICU and 43 cases ( 29 males, 14 females) underwent lumbar puncture after death. The mean age was 56.5 years (range 20-84) and the mean length of stay in ICU was 19.3days (range 5-94). Nine patients were excluded from the study because we could not obtain consent for LP in four of them and four other patients expired during their first $24 \mathrm{~h}$ of admission in ICU.

The last excluded patient was a man with the diagnosis of acute bacterial meningitis. The mean interval from death to performing LP was approx 9 min (range 4-15); $55.8 \%(N=24)$ of the patients had fever during their hospital course and $4.7 \%(N=2)$ had neck stiffness on physical examination. All of the patients had received different types of antibiotics in their treatments.
CSF was completely normal (protein less than $45 \mathrm{mg} /$ dl, WBC less than $5 / \mathrm{ml}$, and glucose concentration more than $60 \%$ of concomitant blood glucose) in $62.8 \%(\mathrm{~N}=$ 27 ) of the patients and we could find at least one abnormal parameter in other cases. Samples compatible with meningitis in three parameters (glucose, protein, and leukocyte counts) were seen in two patients.

Lumbar puncture had not been performed in these two patients and they had received antibiotics with impression of sepsis. Eight patients had been admitted with this impression. There was no positive CSF culture as all the patients were receiving different types of antibiotics before death.

\section{Discussion}

Adequate and proper treatment of infections among ICU-admitted patients appears to be an important factor for improving prognosis. Meningitis is one of the causes of nosocomial infections in ICU and may be missed in such patients. Adelson-Mitty et al. ${ }^{[2]}$ showed that LP is a low-yield procedure in evaluation of fever especially for diagnosis of bacterial meningitis in surgical patients who admitted to ICU without history of head trauma or a neurosurgical operation.

In our study CSF parameters compatible with meningitis were found in two patients, they were receiving antibiotics with impression of sepsis due to pneumonia and urinary tract infections respectively, and LP had not been done for them before death (Table 1).

These two patients are not statistically significant $(P=1)$ but considering a group of patients who admitted to ICU with impression of sepsis, these findings are significant regarding Fisher exact test $(P=0.04)$.

There was no significant difference in surgical and medical cases $(P=0.6)$.

We suggest that meningitis should be considered in patients who admitted to ICU with impression of sepsis

\begin{tabular}{llllllll}
\hline \multicolumn{2}{l}{ Table 1: Characteristic of patients with diagnosis of meningitis } \\
\hline Age & Sex & Cause of hospital admission & Cause of ICU admission & CSF WBC & CSF protein & CSF sugar & History of surgery \\
84 & Male & Colon tumor & Sepsis & 250 & 200 & 10 & Yes \\
35 & Female & UTlsepsis & sepsis & 100 & 68 & 35 & No \\
\hline
\end{tabular}


even in cases that another source for infection is found.

Although the incidence of nosocomial meningitis is low in a neurology ICU but considering its poor prognosis with inappropriate treatment, necessitates more liberal use of LP in ICU-admitted patients especially with impression of sepsis. ${ }^{[3]}$

There are several limitations to our study, as all the patients were receiving antibiotics; we could not find any positive blood or CSF cultures. Another limitation is that our sample did not include a large number of patients and we also did not investigate the possibility of viral, fungal, or other atypical causes of meningitis.

In spite of these limitations our findings support the view that meningitis may be missed and LP will be in- formative in evaluation of septic patients during admission in ICU. More expanded studies with performing LP in these cases before death will help to make the best decision for the patients.

\section{References}

1. Marin HK, Glenda S, Suzanne W, Victoria JF. Inadequate antimicrobial treatment of infections a risk factor for hospital mortality among critically ill patients. Chest 1999;115:462-74.

2. Adelson-Mitty J, Fink MP, Lisbon A. The value of lumbar puncture in the evaluation critically ill, non-immunosuppressed, surgical patients: a retrospective analysis of 70 cases. Intens Care Med 1997;23:749-52.

3. Dettenkofer M, Ebner W, Els T, Babikir R, Lucking C, Pelz K, et al. Surveillance of nosocomial infections in a neurology intensive care unit. J Neurol 2001;248:959-64. 